\title{
Hydrodynamics of Spatially Ordered Superfluids
}

\author{
H.T.C. Stoof, ${ }^{1}$ Kieran Mullen, ${ }^{2}$ Mats Wallin, ${ }^{3}$ and S.M. Girvin ${ }^{4}$ \\ ${ }^{1}$ University of Utrecht, Institute for Theoretical Physics, Princetonplein 5, \\ P.O. Box 80.006, 3508 TA Utrecht, The Netherlands \\ ${ }^{2}$ University of Oklahoma, Department of Physics, Norman, OK 73019-0225 \\ ${ }^{3}$ Royal Institute of Technology, Department of Theoretical Physics, S-100 44 Stockholm, Sweden \\ ${ }^{4}$ Indiana University, Department of Physics, Bloomington, IN 47405
}

\begin{abstract}
We derive the hydrodynamic equations for the supersolid and superhexatic phases of a neutral two-dimensional Bose fluid. We find, assuming that the normal part of the fluid is clamped to an underlying substrate, that both phases can sustain third-sound modes and that in the supersolid phase there are additional modes due to the superfluid motion of point defects (vacancies and interstitials).
\end{abstract}

PACS numbers: $67.70 .+\mathrm{n}, 67.80 .-\mathrm{s}$ 


\section{INTRODUCTION}

Liquid helium $\left({ }^{4} \mathrm{He}\right)$ has a reputation for being the first substance in which one is able to observe many macroscopic quantum phenomena. In particular, it was the first system that could sustain superfluid flow, 1 and as a consequence display a number of amazing properties such as second sound, quantized vortices and the fountain effect. Furthermore, thin superfluid helium films were the first two-dimensional systems experimentally proven to undergo a Kosterlitz-Thouless transition to the normal state.2 More recently it may have been observed 3 that on weakly-binding substrates these films are the first-known spatially ordered superfluids 1

More precisely, measurements of the third-sound resonance frequency (which is proportional to the square root of the superfluid density) of submonolayer helium films on hydrogen and deuterium substrates apparently indicate two independent Kosterlitz-Thouless transitions: the usual superfluid to normal transition at a temperature $T_{K T}$ that obeys the expected universal jump relation, 目 and a second new transition at a temperature $T_{c}$ which is roughly $0.5 T_{K T}$ for all coverages. The second transition appears as a sharp (but not discontinuous) rise or dip in the superfluid density depending on the substrate.

In an attempt to explain these experimental results we have recently proposed that below the second critical temperature the superfluid helium film is in a spatially ordered phase exhibiting both off-diagonal (superfluid) and diagonal (hexatic) long-range order in the

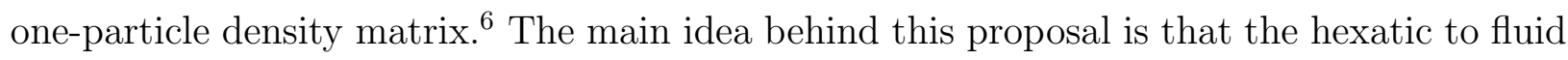
transition is known to be a Kosterlitz-Thouless transition driven by disclination unbinding. 0 (Disclinations are defects in the orientational order of a crystal created by the insertion or removal of a wedge of atoms, as shown in Fig. 1.) Therefore, our physical picture of the experiments is that at sufficiently low temperatures the film is in a superhexatic phase with only a dilute gas of bound vortices and bound disclinations present due to thermal fluctuations. For entropic reasons the disclinations then unbind at $T_{c}$, leading to a transition from a superhexatic to a superfluid phase since the vortices remain bound at this transition 
and the presence of free disclinations destroys the hexatic long-range order. At $T_{K T}$ the vortices then also unbind and the film is finally forced into the normal liquid phase.

Of course, to make sure that the above picture is qualitatively correct we must also consider the interaction between vortices and disclinations. This is even more pressing if one realizes that in a supersolid phase (where all disclination pairs are themselves bound into pairs or triples) this interaction is of long range and depends logarithmically on the distance between the two kinds of defects. Fortunately, it turns out that this is no longer true in the superhexatic phase due to the screening of the interaction by the surrounding gas of disclination pairs. A renormalization-group analysis actually shows that the vortexdisclination interaction is irrelevant and that the two separate Kosterlitz-Thouless transitions indeed survive. Nevertheless, the superfluid density is influenced in a non-universal way by the unbinding of the disclinations and Monte-Carlo simulations even show that on the basis of our hypothesis a rough qualitative agreement with the experiments of Chen and Mochel can be obtained.

However, to definitely identify the phase below $T_{c}$ more detailed information is needed. As a first step towards this goal we here present the two-dimensional hydrodynamic equations of a superhexatic by describing the superhexatic as a supersolid with free dislocations (i.e. disclination pairs ). As a result of this approach we will also be able to consider the hydrodynamics of the supersolid phase, for which there is at present a renewed interest both in the context of Josephson-junction arrays ${ }^{8}$ and solid ${ }^{4}$ He. ${ }^{3}$ Moreover, spatially ordered superfluid states have recently been proposed to be also relevant for the fractional quantum Hall effect, 10 since this effect can be understood as a condensation of composite bosons.11 We therefore believe that the methods developed below might, if extended to bosons interacting with a Chern-Simons gauge field, also be used to obtain a description of the dynamics of such exotic quantum Hall states.

We have organized the paper in the following manner. In Sec. II] we first consider the normal solid and hexatic phases by formulating a gauge theory that describes the phonons, the dislocations and the interaction between them. From this theory we then deduce in Sec. 
III] for both phases the dynamics of the appropriate hydrodynamic degrees of freedom. In Sec. $\mathbb{V}$ we incorporate the effects of the additional superfluid order parameter ${ }^{12}$ into the hydrodynamic equations derived in Sec. [IT and discuss the various long-wavelength modes in the supersolid and superhexatic phases obtained in this manner. We conclude in Sec. $\square$ with a discussion on the possible relevance of our work to future experiments on submonolayer helium films and with a physical interpretation of our results.

\section{GAUGE THEORY OF PHONONS AND DISLOCATIONS}

In this section we will derive the long-wavelength (quantum) dynamics of the solid and hexatic phases. The discussion closely follows work by Kleinert, 13 save that we will not include higher gradient elasticity. This leads to a considerable simplification of the theory but implies that we cannot properly treat the dynamics of the disclinations. Fortunately, for our purposes only the dynamics of the dislocations is of importance and this simplification is justified.

\section{A. Solid}

In the case of an isotropic crystal, the action for the displacement field $u_{i}(\vec{x}, \tau)$ in the presence of a pair of dislocations is given by 13

$$
S\left[u_{i}\right]=\int_{0}^{\hbar \beta} d \tau \int d \vec{x}\left\{\frac{\rho}{2}\left(\partial_{\tau} u_{i}-\beta_{i}\right)^{2}+\mu\left(u_{i j}-\frac{\beta_{i j}+\beta_{j i}}{2}\right)^{2}+\frac{\lambda}{2}\left(u_{i i}-\beta_{i i}\right)^{2}\right\},
$$

where $u_{i j}=\left(\partial_{i} u_{j}+\partial_{j} u_{i}\right) / 2$ is the strain tensor, $\mu$ and $\lambda$ are the usual Lamé coefficients 14 and $\rho$ is the average mass density. The unphysical (and singular) contributions arising from the multivaluedness of $u_{i}(\vec{x}, \tau)$ are compensated by the quantities $\beta_{j}$ and $\beta_{i j}$ (also known as the 'plastic distortion'). Their relationship to the defects is best explained by the Volterra construction. 15 Let $\mathcal{C}$ be a small loop bounding a section of two dimensional crystal that is excised from the whole (cf. Fig. 2). The edges of the loop are drawn together and form a line $\mathcal{L}$. This line may be time dependent, and its definition is not unique. However, the 
topological defects (i.e. two dislocations with opposite Burgers' vectors) associated with the distortion of the surface are always located at the endpoints of $\mathcal{L}$. If $\pm \vec{B}$ are the Burgers' vectors of the dislocations constituting the pair and if $\vec{v}$ is their velocity then $\beta_{i j}=\delta_{i}(\mathcal{L}) B_{j}$ and $\beta_{j}=-v_{i} \delta_{i}(\mathcal{L}) B_{j}$. The delta function $\delta_{i}(\mathcal{L})$ is singular on the time-dependent Volterra cutting line $\mathcal{L}$ of the dislocations and is directed along the normal vector. If the cutting line $\mathcal{L}$ is parameterized by $\vec{x}(s, \tau)$ with $0 \leq s \leq 1$, this means mathematically that

$$
\delta_{i}(\mathcal{L})=-\epsilon_{i j} \int_{0}^{1} d s \frac{\partial x_{j}(s, \tau)}{\partial s} \delta(\vec{x}-\vec{x}(s, \tau)),
$$

where $\epsilon_{i j}$ is the two-dimensional antisymmetric tensor. Note that the dislocations are assumed to be able to move freely, without any friction, through the crystal because the equations of motion for the displacement field allow for time-dependent solutions that precisely correspond to such evolutions of the crystal. 1 We will come back to the issue of friction in Sec. III when we consider the effects of dissipation.

We now first perform a Hubbard-Stratonovich transformation by introducing the auxillary variable $\vec{p}$ (representing the momentum density) and adding the quadratic term

$$
\int_{0}^{\hbar \beta} d \tau \int d \vec{x} \frac{1}{2 \rho}\left(p_{i}-i \rho\left(\partial_{\tau} u_{i}-\beta_{i}\right)\right)^{2}
$$

to the action, which may now be rewritten as

$$
\begin{aligned}
S\left[p_{i}, u_{i}\right]=\int_{0}^{\hbar \beta} d \tau \int d \vec{x}\left\{\frac{p_{i}^{2}}{2 \rho}\right. & +\mu\left(u_{i j}-\frac{\beta_{i j}+\beta_{j i}}{2}\right)^{2} \\
& \left.+\frac{\lambda}{2}\left(u_{i i}-\beta_{i i}\right)^{2}-i p_{i}\left(\partial_{\tau} u_{i}-\beta_{i}\right)\right\} .
\end{aligned}
$$

Integrating out $\vec{p}$ would return the original action up to an unimportant constant. In a similar manner we then also introduce the symmetric stress tensor $\sigma_{i j}$, to decouple the terms quadratic in the strain. This results in

$$
\begin{aligned}
S\left[p_{i}, \sigma_{i j}, u_{i}\right]=\int_{0}^{\hbar \beta} d \tau \int d \vec{x}\left\{\frac{p_{i}^{2}}{2 \rho}\right. & +\frac{1}{4 \mu}\left(\sigma_{i j}^{2}-\frac{\nu}{1+\nu} \sigma_{i i}^{2}\right) \\
& \left.-i p_{i}\left(\partial_{\tau} u_{i}-\beta_{i}\right)+i \sigma_{i j}\left(u_{i j}-\frac{\beta_{i j}+\beta_{j i}}{2}\right)\right\},
\end{aligned}
$$


with $\nu=\lambda /(2 \mu+\lambda)$. The partition function is now given by the functional integral

$$
Z=\int d\left[p_{i}\right] \int d\left[\sigma_{i j}\right] \int d\left[u_{i}\right] \exp \left\{-\frac{1}{\hbar} S\left[p_{i}, \sigma_{i j}, u_{i}\right]\right\}
$$

where the integration over $\sigma_{i j}$ is only over the symmetrical part since we have not included higher gradient elasticity.

We can now perform the integration over the displacement field. Because the action is linear in $u_{i}$ this simply leads to the constraint

$$
\partial_{\tau} p_{j}=\partial_{i} \sigma_{i j}
$$

This constraint can be automatically satisfied if we introduce the vector field $A_{j}$ and the tensor field $A_{i j}$ by setting $\sigma_{i j}=\epsilon_{i k} \partial_{k} A_{j}+\epsilon_{k i} \partial_{\tau} A_{k j}$ and $p_{j}=\epsilon_{k i} \partial_{i} A_{k j}$. Substituting these relations into the action we find that the interaction between the gauge fields (i.e. the phonons) and the dislocations is given by

$$
S_{i n t}\left[A_{i j}, A_{j}\right]=\int_{0}^{\hbar \beta} d \tau \int d \vec{x}\left\{-i A_{i} \alpha_{i}+i A_{i j} J_{i j}\right\}
$$

where after several partial integrations the unphysical singularities of $\beta_{i}$ and $\beta_{i j}$ have disappeared and only the dislocation density and the dislocation current density remain. Introducing also the function $\delta(\mathcal{P})=\delta(\vec{x}(1, \tau))-\delta(\vec{x}(0, \tau))$, which denotes the difference between a delta function at one end of the cutting line $\mathcal{L}$ and a delta function at the other end, these densities and currents can conveniently be written as $\alpha_{j}=\delta(\mathcal{P}) B_{j}$ and $J_{i j}=-v_{i} \delta(\mathcal{P}) B_{j}$, respectively. As a direct consequence of the above definitions they obey the conservation law

$$
\partial_{\tau} \alpha_{j}=\partial_{i} J_{i j}
$$

In addition, the dynamics of the phonons is determined by the remaining quadratic terms in the action which expressed in terms of the gauge fields $A_{j}$ and $A_{i j}$ yield

$$
S_{0}\left[A_{i j}, A_{j}\right]=\int_{0}^{\hbar \beta} d \tau \int d \vec{x}\left\{\frac{\left(\epsilon_{i k} \partial_{k} A_{i j}\right)^{2}}{2 \rho}+\frac{1}{4 \mu}\left(\sigma_{i j}^{2}-\frac{\nu}{1+\nu} \sigma_{i i}^{2}\right)\right\}
$$


with $\sigma_{i j}$ equal to $\epsilon_{i k}\left(\partial_{k} A_{j}-\partial_{\tau} A_{k j}\right)$. Comparing this result with Eq. (11) we observe that the stress and the physical part of the strain $u_{i j}^{P h y s} \equiv u_{i j}-\left(\beta_{i j}+\beta_{j i}\right) / 2$ are related by $\sigma_{i j}=2 \mu u_{i j}^{P h y s}+\lambda \delta_{i j} u_{k k}^{P h y s}$ and therefore by

$$
u_{i j}^{P h y s}=\frac{1}{2 \mu}\left(\sigma_{i j}-\frac{\nu}{1+\nu} \delta_{i j} \sigma_{k k}\right) .
$$

We will have need of the latter relation in Sec. III, when we discuss hydrodynamics. A more formal way to justify it is to add to the action $S\left[u_{i}\right]$ a source term

$$
\int_{0}^{\hbar \beta} d \tau \int d \vec{x} K_{i j}\left(u_{i j}-\frac{\beta_{i j}+\beta_{j i}}{2}\right)=\int_{0}^{\hbar \beta} d \tau \int d \vec{x} K_{i j} u_{i j}^{P h y s}
$$

and perform the same manipulations as before. We then find that the source $K_{i j}$ indeed couples linearly to the right-hand side of Eq. (10).

Following Kleinert, we now notice that the above theory has a gauge symmetry as a result of the fact that the gauge fields $A_{i}$ and $A_{i j}$ are not uniquely determined if the stresses $\sigma_{i j}$ and momenta $p_{i}$ are known. Indeed, $\sigma_{i j}$ and $p_{i}$ are invariant under the gauge transformation $A_{i} \rightarrow A_{i}+\partial_{\tau} \Lambda_{i}$ and $A_{i j} \rightarrow A_{i j}+\partial_{i} \Lambda_{j}$. Hence $S_{0}\left[A_{i j}, A_{j}\right]$ is also invariant. Moreover, due to the conservation law in Eq. (8), the interaction $S_{\text {int }}\left[A_{i j}, A_{j}\right]$ is invariant too.

To calculate the partition function we therefore need some gauge-fixing procedure. The symmetry of $\sigma_{i j}$ requires that

$$
\epsilon_{i j} \sigma_{i j}=\partial_{j} A_{j}-\partial_{\tau}\left(A_{j j}\right)=0
$$

We would now like to write the gauge fields as the appropriate derivatives of unconstrained fields. Using the above gauge symmetry we can always take $A_{i}=\epsilon_{i j} \partial_{j} \chi$ and $A_{i i}=0$. This, however, does not completely fix the gauge because these conditions are still invariant under the smaller group of transformations $\chi \rightarrow \chi+\partial_{\tau} \Lambda$ and $A_{i j} \rightarrow A_{i j}+\partial_{i}\left(\epsilon_{j k} \partial_{k} \Lambda\right)$. To see more clearly the consequences of this residual symmetry we expand $A_{i j}$ into its longitudinal and transverse components (with respect to both indices), i.e.

$$
A_{i j}=\partial_{i}\left(\partial_{j} A^{L L}\right)+\partial_{i}\left(\epsilon_{j k} \partial_{k} A^{L T}\right)+\epsilon_{i k} \partial_{k}\left(\partial_{j} A^{T L}\right)+\epsilon_{i k} \partial_{k}\left(\epsilon_{j l} \partial_{l} A^{T T}\right),
$$


where we have introduced four new fields. The tracelessness of $A_{i j}$ can then be fulfilled by taking $A^{L L}=-A^{T T}$. In addition, the residual gauge symmetry can now be written as $\chi \rightarrow \chi+\partial_{\tau} \Lambda$ and $A^{L T} \rightarrow A^{L T}+\Lambda$. This shows that instead of the fields $\chi$ and $A^{L T}$ we must use the gauge-invariant field $\chi^{\prime} \equiv \chi-\partial_{\tau} A^{L T}$ together with $\Lambda$ as integration variables. The associated change of measure can be incorporated in the normalization and the same is true for the 'volume' $\int d[\Lambda]$ of the residual gauge group because the action is gauge invariant and therefore cannot depend on $\Lambda$. After this gauge-fixing procedure the partition function thus becomes

$$
Z=\int d\left[A^{T T}\right] \int d\left[A^{T L}\right] \int d\left[\chi^{\prime}\right] \exp \left\{-\frac{1}{\hbar}\left(S_{0}\left[A^{T T}, A^{T L}, \chi^{\prime}\right]+S_{i n t}\left[A^{T T}, A^{T L}, \chi^{\prime}\right]\right)\right\}
$$

Note that we are left with three physical degrees of freedom, which is the correct number in two dimensions since $\sigma_{i j}$ and $p_{i}$ contain in principle a total of five degrees of freedom but we have two constraints in Eq. (6). Note also that the transformation from $\sigma_{i j}$ and $p_{i}$ to $A^{T T}$, $A^{T L}$ and $\chi^{\prime}$ is a linear one so that the Jacobian involved in the calculation of the partition function is simply an unimportant constant. In particular, the stress is given by

$$
\sigma_{i j}=\epsilon_{i k} \epsilon_{j \ell} \partial_{k} \partial_{\ell} \chi^{\prime}+\partial_{\tau}\left(\partial_{i} \partial_{j} A^{T L}+\epsilon_{i k} \partial_{k} \partial_{j} A^{T T}+\epsilon_{j k} \partial_{k} \partial_{i} A^{T T}\right)
$$

which is manifestly symmetric in $i$ and $j$.

A straightforward calculation now shows that the free part of the action is

$$
\begin{aligned}
S_{0}\left[A^{T T}, A^{T L}, \chi^{\prime}\right] & =\int_{0}^{\hbar \beta} d \tau \int d \vec{x}\left\{\frac{1}{2 \mu}\left(\partial_{\tau} \partial^{2} A^{T T}\right)^{2}+\frac{1}{2 \rho}\left(\partial_{i} \partial^{2} A^{T T}\right)^{2}\right. \\
& +\frac{1}{4 \mu(1+\nu)}\left(\partial_{\tau} \partial^{2} A^{T L}\right)^{2}+\frac{1}{2 \rho}\left(\partial_{i} \partial^{2} A^{T L}\right)^{2}+\frac{1}{4 \mu(1+\nu)}\left(\partial^{2} \chi^{\prime}\right)^{2} \\
& \left.-\frac{1}{2 \mu} \frac{\nu}{1+\nu}\left(\partial_{\tau} \partial^{2} A^{T L}\right)\left(\partial^{2} \chi^{\prime}\right)\right\}
\end{aligned}
$$

It contains four modes: The part involving $A^{T T}$ has a pair of modes (corresponding to $\pm \vec{k}$ ) with $\omega^{2}=\mu \vec{k}^{2} / \rho$. These modes therefore represent the transverse phonons with a speed of sound $\sqrt{\mu / \rho}$. The part involving $A^{T L}$ and $\chi^{\prime}$ has another pair of modes with a dispersion obeying $\omega^{2}=(2 \mu+\lambda) \vec{k}^{2} / \rho$. These represent the longitudinal phonons with a speed of 
sound $\sqrt{(2 \mu+\lambda) / \rho}$. Interestingly, these results can be understood much more easily if we introduce the field

$$
\chi^{\prime \prime} \equiv \chi^{\prime}-\nu \partial_{\tau} A^{T L}
$$

since then the above action becomes

$$
\begin{aligned}
S_{0}\left[A^{T T}, A^{T L}, \chi^{\prime \prime}\right] & =\int_{0}^{\hbar \beta} d \tau \int d \vec{x}\left\{\frac{1}{2 \mu}\left(\partial_{\tau} \partial^{2} A^{T T}\right)^{2}+\frac{1}{2 \rho}\left(\partial_{i} \partial^{2} A^{T T}\right)^{2}\right. \\
& \left.+\frac{1-\nu}{4 \mu}\left(\partial_{\tau} \partial^{2} A^{T L}\right)^{2}+\frac{1}{2 \rho}\left(\partial_{i} \partial^{2} A^{T L}\right)^{2}+\frac{1}{4 \mu(1+\nu)}\left(\partial^{2} \chi^{\prime \prime}\right)^{2}\right\},
\end{aligned}
$$

so that the fields are completely uncoupled. Notice that the $\chi^{\prime \prime}$ field has no kinetic term, which explains why we obtained above only four modes instead of six, as might have been expected in first instance.

Furthermore, if we introduce the usual Burgers' field $\vec{b}(\vec{x}, \tau)$ for the total dislocation density, which is nothing more than the sum of the density $\alpha_{i}$ over all dislocation pairs, then the interaction with the dislocations acquires the form

$$
S_{i n t}\left[A^{T T}, A^{T L}, \chi^{\prime}\right]=\int_{0}^{\hbar \beta} d \tau \int d \vec{x} i\left\{\chi^{\prime} \epsilon_{i j} \partial_{j} b_{i}-A^{T T} \partial_{\tau} \partial_{i} b_{i}\right\}
$$

where we have made use of Eq. (8) to express the longitudinal part of the current density $J_{i j}$ in terms of the time derivative of $b_{i}$. Decomposing $\vec{b}$ into its transverse and longitudinal parts, i.e. $b_{i}=\partial_{i} b^{L}+\epsilon_{i j} \partial_{j} b^{T}$, the interaction finally becomes

$$
S_{\text {int }}\left[A^{T T}, A^{T L}, \chi^{\prime}\right]=\int_{0}^{\hbar \beta} d \tau \int d \vec{x} i\left\{\chi^{\prime} \partial^{2} b^{T}-A^{T T} \partial_{\tau} \partial^{2} b^{L}\right\}
$$

The total action $S=S_{0}+S_{\text {int }}$ reduces for time-independent configurations to the one we previously used for a discussion of the critical properties of the superhexatic.6 Integrating out the fields $A^{T T}$ and $\chi^{\prime}$ we can now find the time-dependent interaction among the dislocations. Physically, these interactions are thus associated with phonon exchange and the time dependence arises due to the finite speeds of sound. This picture also explains why the effective action for $\chi^{\prime}$ contains just one pair of modes: The self-interaction of the transverse dislocation density can only be mediated by longitudinal phonons. 


\section{B. Hexatic}

Up to this point the dislocation density has not been an independent dynamical variable, since we have specified the positions of the dislocations at all times and thus neglected the influence of the phonon dynamics on their motion. However, to describe the hexatic phase we want to integrate out the dislocations in the plasma (or continuous) approximation. For that we need the free action of the field $\vec{b}$. Here we can again make use of the results obtained by Kleinert, who showed that the energy associated with the nonlinear stresses at the heart of the defect can be lumped into a 'core contribution' to the action.13.17 In our notation this contribution becomes

$$
\begin{aligned}
S_{0}\left[b_{i}\right] & =\int_{0}^{\hbar \beta} d \tau \int d \vec{x} \frac{E_{c}}{2} b_{i}\left(\frac{\rho}{\mu} \frac{\partial_{\tau}^{2}}{\partial^{2}}+1\right) b_{i} \\
& =\int_{0}^{\hbar \beta} d \tau \int d \vec{x} \frac{E_{c}}{2}\left\{b^{T}\left(\frac{\rho}{\mu} \partial_{\tau}^{2}+\partial^{2}\right) b^{T}+b^{L}\left(\frac{\rho}{\mu} \partial_{\tau}^{2}+\partial^{2}\right) b^{L}\right\} .
\end{aligned}
$$

This action represents free propagation of the dislocation density fluctuations which, as

mentioned previously, are permitted by the classical equations of motion 16 and neglects dissipative coupling of the dislocation cores to the phonons.

Integrating out the Burgers' field using Eqs. (19) and (20), we obtain the following results. The effective action for $A^{T T}$ becomes

$$
\begin{aligned}
S^{e f f}\left[A^{T T}\right]=\int_{0}^{\hbar \beta} d \tau \int d \vec{x}\{ & \frac{1}{2 \mu}\left(\partial_{\tau} \partial^{2} A^{T T}\right)^{2}+\frac{1}{2 \rho}\left(\partial_{i} \partial^{2} A^{T T}\right)^{2} \\
& \left.+\frac{1}{2 E_{c}}\left(\partial_{\tau} \partial_{i} A^{T T}\right)\left(\frac{\rho}{\mu} \frac{\partial_{\tau}^{2}}{\partial^{2}}+1\right)^{-1}\left(\partial_{\tau} \partial_{i} A^{T T}\right)\right\} .
\end{aligned}
$$

As shown in Fig. 3a, it contains two pairs of modes which for $\vec{k}^{2} \gg \mu / 2 E_{c}$ all have a dispersion obeying $\omega^{2} \simeq \mu \vec{k}^{2} / \rho$. However, for $\vec{k}^{2} \ll \mu / 2 E_{c}$, one pair of modes has a dispersion $\omega^{2} \simeq$ $\mu^{2} / E_{c} \rho+2 \mu \vec{k}^{2} / \rho$ with a gap whereas the other pair of modes is gapless with $\omega^{2} \simeq 2 E_{c} \vec{k}^{4} / \rho$. This is consistent with our expectation that in the hexatic phase there should only be one pair of transverse gapless modes with a softer dispersion than that of the transverse phonon modes in a true solid. 
The effective action for $\chi^{\prime}$ and $A^{T L}$ in the hexatic phase is

$$
\begin{aligned}
S^{e f f}\left[A^{T L}, \chi^{\prime}\right] & =\int_{0}^{\hbar \beta} d \tau \int d \vec{x}\left\{\frac{1}{4 \mu(1+\nu)}\left(\partial_{\tau} \partial^{2} A^{T L}\right)^{2}+\frac{1}{2 \rho}\left(\partial_{i} \partial^{2} A^{T L}\right)^{2}\right. \\
& +\frac{1}{4 \mu(1+\nu)}\left(\partial^{2} \chi^{\prime}\right)^{2}-\frac{1}{2 \mu} \frac{\nu}{1+\nu}\left(\partial_{\tau} \partial^{2} A^{T L}\right)\left(\partial^{2} \chi^{\prime}\right) \\
& \left.+\frac{1}{2 E_{c}}\left(\partial_{i} \chi^{\prime}\right)\left(\frac{\rho}{\mu} \frac{\partial_{\tau}^{2}}{\partial^{2}}+1\right)^{-1}\left(\partial_{i} \chi^{\prime}\right)\right\}
\end{aligned}
$$

Integrating out also $A^{T L}$ we finally arrive at the effective action for $\chi^{\prime}$. It reads

$$
\begin{aligned}
S^{e f f}\left[\chi^{\prime}\right]=\int_{0}^{\hbar \beta} d \tau \int d \vec{x}\{ & \frac{1}{4 \mu(1+\nu)}\left(\partial^{2} \chi^{\prime}\right)^{2}+\frac{1}{2 E_{c}}\left(\partial_{i} \chi^{\prime}\right)\left(\frac{\rho}{\mu} \frac{\partial_{\tau}^{2}}{\partial^{2}}+1\right)^{-1}\left(\partial_{i} \chi^{\prime}\right) \\
& \left.+\frac{1}{2}\left(\frac{1}{2 \mu} \frac{\nu}{1+\nu}\right)^{2}\left(\partial_{\tau} \partial^{2} \chi^{\prime}\right)\left(\frac{\partial_{\tau}^{2}}{2 \mu(1+\nu)}+\frac{\partial^{2}}{\rho}\right)^{-1}\left(\partial_{\tau} \partial^{2} \chi^{\prime}\right)\right\}
\end{aligned}
$$

and also contains two pairs of modes (cf. Fig. 3b). For $\vec{k}^{2} \gg \mu / 2 E_{c}$ we recover of course the ordinary sound dispersions $\omega^{2} \simeq 2 \mu \overrightarrow{k^{2}} /(\rho(1-\nu))=(2 \mu+\lambda) \vec{k}^{2} / \rho$ and $\omega^{2} \simeq \mu \vec{k}^{2} / \rho$. However, in the limit $\vec{k}^{2} \ll \mu / 2 E_{c}$ these evolve into a pair of gapped modes with $\omega^{2} \simeq 2 \mu^{2} /\left(E_{c} \rho(1-\nu)\right)$ and a pair of propagating modes with $\omega^{2} \simeq 2 \mu(1+\nu) \vec{k}^{2} / \rho$, respectively. Clearly, the same mode structure is also present in the effective action for $A^{T L}$ (obtained by integrating out $\chi^{\prime}$ instead of $A^{T L}$ ) which indicates that in the hexatic phase the longitudinal velocity is renormalized downwards to $\sqrt{2 \mu(1+\nu) / \rho}$.

\section{HYDRODYNAMICS OF SPATIALLY ORDERED PHASES}

We now turn to the linear hydrodynamics of the solid and hexatic phases that follows from the theory presented above. For the sake of clarity, and because it will turn out to be less important for our purposes, we will not discuss temperature fluctuations in the following. However, having derived the relevant energy densities in Secs. ПA and ПВ it is in principle straightforward to include temperature fluctuations in our theory and, in particular, to arrive at the extension of the hydrodynamic equations presented below that is required if one wants to consider also the hydrodynamic mode due to energy conservation. After the equations of motion for the hydrodynamic variables are determined, we can find the propagating and 
diffusive modes. This is done as before, by Fourier transforming the equations of motion and determining the dispersion $\omega(k)$. Propagating modes appear as complex roots of a characteristic equation and will always occur in pairs. Each physically distinct propagating excitation such as longitudinal or transverse sound corresponds therefore to two roots or modes.

We start by considering the mass-density fluctuation $\delta \rho$ above the average mass density $\rho$ and initially neglect the possible presence of vacancies and interstitials. To lowest order in the strain, the density fluctuation equals $-\rho u_{i i}^{P h y}$ so up to that order we obtain

$$
\partial_{\tau} \delta \rho=-\rho \partial_{\tau} u_{i i}^{\text {Phys }}=-\frac{\rho}{2(\mu+\lambda)} \partial_{\tau} \sigma_{i i}
$$

if we make use of Eq. (10) to relate the stress and the strain. Using also the decomposition $\sigma_{i i}=\partial^{2} \chi^{\prime}+\partial_{\tau} \partial^{2} A^{T L}$ from Eq. (14) we may write this as a pair of continuity equations

$$
\begin{gathered}
\partial_{\tau} \delta \rho=\partial_{i} g_{i}^{L}, \\
\partial_{\tau} g_{j}^{L}=\partial_{i} \pi_{i j}^{D},
\end{gathered}
$$

where the longitudinal momentum density is given by

$$
g_{i}^{L}=-\frac{\rho}{2(\mu+\lambda)} \partial_{\tau} \partial_{i}\left(\chi^{\prime}+\partial_{\tau} A^{T L}\right)
$$

and the diagonal part of the stress tensor by

$$
\pi_{i j}^{D}=-\frac{\rho \delta_{i j}}{2(\mu+\lambda)} \partial_{\tau}^{2}\left(\chi^{\prime}+\partial_{\tau} A^{T L}\right)
$$

In the absence of defects, the hydrodynamic quantities $g_{i}^{L}$ and $\pi_{i j}^{D}$ are precisely equal to the longitudinal part of $p_{i}$ and the diagonal part of $\sigma_{i j}$, respectively. This can be seen in the following manner. In an ideal solid we have no defects, and variation of the action in Eq. (17) gives $\chi^{\prime \prime}=0$ or $\chi^{\prime}=\nu \partial_{\tau} A^{T L}$, which we may use to eliminate $\chi^{\prime}$. The equation of motion for $A^{T L}$ generated in this way is

$$
\partial_{\tau}^{2} A^{T L}=-\frac{2 \mu+\lambda}{\rho} \partial^{2} A^{T L} .
$$


If we substitute this back into the definitions of $g_{i}^{L}$ and $\pi_{i j}^{D}$ we obtain the longitudinal part of $p_{i}$ and the diagonal part of $\sigma_{i j}$ as given in section $\mathbb{1 1}$. In the presence of defects with their own dynamics this is no longer true, since the dislocation density couples to the gauge fields and alters the equations of motion. To avoid confusion about this point we have, therefore, introduced a new notation for the hydrodynamic momentum density and stress tensor which we will use for the rest of the paper.

We also note that the above equations are not Galilean invariant and are therefore only valid in a specific reference frame. This is a result of the fact that the gauge theory of Sec. [1] has implicitly used the existence of an ideal lattice with respect to which the displacements $\vec{u}(\vec{x}, \tau)$ are defined. 15 Hence, the prefered reference frame corresponds to that frame in which this ideal lattice is at rest. This is the case for all the hydrodynamic equations presented below.

In this ideal solid without interstitials or vacancies the pressure fluctuation (following from $\left.\pi_{i j}^{D}=-\delta_{i j} \delta p\right)$ equals

$$
\delta p=\frac{\rho}{2(\mu+\lambda)}(1+\nu) \partial_{\tau}^{3} A^{T L}=\frac{\rho}{2 \mu+\lambda} \partial_{\tau}^{3} A^{T L}
$$

and the mass-density fluctuation becomes

$$
\delta \rho=-\frac{\rho}{2(\mu+\lambda)}(1+\nu) \partial_{\tau} \partial^{2} A^{T L}=-\frac{\rho}{2 \mu+\lambda} \partial_{\tau} \partial^{2} A^{T L}
$$

Substituting the equation of motion Eq. (28) for $A^{T L}$ into Eq. (29), we obtain the desired constitutive equation

$$
\delta p=\frac{2 \mu+\lambda}{\rho} \delta \rho
$$

Together with the hydrodynamic equations (25) this correctly leads to the sound equation

$$
\partial_{\tau}^{2} \delta \rho=-\frac{2 \mu+\lambda}{\rho} \partial^{2} \delta \rho=-c_{\|}^{2} \partial^{2} \delta \rho,
$$

with $c_{\|}$the longitudinal sound velocity.

However, as stressed first by Martin, Parodi, and Pershan 18 and again by Zippelius, Halperin, and Nelson 19 we are not in general allowed to assume that the crystal is ideal, 
without vacancies or interstitials. We must include the effects of (long-wavelength) fluctuations in the net defect density $n_{\Delta}$, which is defined as the density of vacancies minus the density of interstitials. To do so we can make use of the fact that in a hexagonal system these defects can be regarded as a 'bound state' of three dislocations with radial Burgers' vectors pointing symmetrically outward (interstitial) or inward (vacancy) 20 This is illustrated for an interstitial in Fig. 4. As a result the interaction of the net defect density with the gauge fields is given by

$$
S_{i n t}\left[A^{T T}, A^{T L}, \chi^{\prime}\right]=\int_{0}^{\hbar \beta} d \tau \int d \vec{x} i \frac{\gamma_{\Delta}}{\mu} n_{\Delta} \partial^{2} \chi^{\prime}
$$

where $V_{0}$ denotes the area deficit induced by a defect in an otherwise perfect crystal and $\gamma_{\Delta}=\mu c_{\|}^{2} V_{0} / 2 c_{\perp}^{2}$. We can verify this result by noting that in the static case (and $n_{\Delta} \rightarrow i n_{\Delta}$ because of our conventions in the imaginary time formalism of Sec. II) the Euler-Lagrange equation for the Airy stress function, following from the action in Eq. (15) together with the above interaction, becomes $\partial^{2} \chi=2(\mu+\lambda) V_{0} n_{\Delta}$ which correctly leads to $\int d \vec{x} u_{i i}^{\text {Phys }}=$ $V_{0} \int d \vec{x} n_{\Delta}$. Furthermore, the free action of the defects becomes (cf. Eq. (20))

$$
S_{0}\left[n_{\Delta}\right]=\int_{0}^{\hbar \beta} d \tau \int d \vec{x} \frac{E_{\Delta}}{2} n_{\Delta}\left(\frac{\rho}{\mu} \frac{\partial_{\tau}^{2}}{\partial^{2}}+1\right) n_{\Delta},
$$

with $E_{\Delta}$ of order $E_{c} V_{0}$.

Redoing our calculations with $n_{\Delta}$ non-zero, we find that $n_{\Delta}$ displaces the $\chi^{\prime}$ field. Therefore $\chi^{\prime \prime}$ in Eq. (16) is also non-zero. Moreover, we now obtain instead of Eq. (32) the coupled set of equations

$$
\begin{gathered}
\partial_{\tau}^{2} \delta \rho=-c_{\|}^{2}\left(1+\frac{\nu \gamma_{\Delta}^{2}}{E_{\Delta} \mu}\right) \partial^{2} \delta \rho+i \gamma_{\Delta}\left(1-\frac{2 \gamma_{\Delta}^{2}}{E_{\Delta} \mu}\right) \partial^{2} n_{\Delta}, \\
\partial_{\tau}^{2} n_{\Delta}=-c_{\perp}^{2}\left(1+\frac{2 \gamma_{\Delta}^{2}}{E_{\Delta} \mu}\right) \partial^{2} n_{\Delta}+i \frac{\gamma_{\Delta} \lambda}{E_{\Delta} \rho^{2}} \partial^{2} \delta \rho,
\end{gathered}
$$

for the longitudinal degrees of freedom. Note that the density fluctuation $\delta \rho$ receives a contribution from both the lattice vibrations as well as from the net defect density, since

$$
\delta \rho=-\frac{1}{c_{\|}^{2}} \partial_{\tau} \partial^{2} A^{T L}+\frac{2 i \gamma_{\Delta}}{c_{\|}^{2}} n_{\Delta}
$$


As a result the longitudinal momentum density has also two contributions

$$
g_{i}^{L}=-\frac{1}{c_{\|}^{2}} \partial_{i} \partial_{\tau}^{2} A^{T L}-\frac{2 i \gamma_{\Delta}}{c_{\|}^{2}} J_{i}^{L},
$$

where $\vec{J}^{L}$ is the longitudinal part of the net defect current density obeying the continuity equation $\partial_{\tau} n_{\Delta}=-\partial_{i} J_{i}^{L}$.

This almost completes our discussion of the hydrodynamical description (without dissipation) of the solid phase. However, we have not yet obtained the transverse modes. From our expressions for the strain tensor $u_{i j}$ one can easily show that in the solid phase the transverse part of the displacement field is given by

$$
u_{i}^{T}=\frac{1}{\mu} \epsilon_{i j} \partial_{j}\left(\partial_{\tau} A^{T T}\right)=\frac{1}{\rho c_{\perp}^{2}} \epsilon_{i j} \partial_{j}\left(\partial_{\tau} A^{T T}\right),
$$

where $c_{\perp}$ is the transverse speed of sound. Hence, the transverse dynamics of the lattice is solely determined by the transverse phonons and we have the additional hydrodynamic equation

$$
\partial_{\tau}^{2} A^{T T}=-c_{\perp}^{2} \partial^{2} A^{T T}
$$

which is completely uncoupled from the previous ones and in particular does not depend on the net defect density $n_{\Delta}$. Moreover, if we introduce the standard hexatic order parameter $\vartheta_{6}$, which is equal to the local bond angle and may therefore be written as

$$
\vartheta_{6} \equiv \frac{1}{2} \epsilon_{i j} \partial_{i} u_{j}=-\frac{1}{2 \rho c_{\perp}^{2}}\left(\partial_{\tau} \partial^{2} A^{T T}\right),
$$

the equation for $A^{T T}$ is equivalent to

$$
\partial_{\tau}^{2} \vartheta_{6}=-c_{\perp}^{2} \partial^{2} \vartheta_{6}
$$

so that $\vartheta_{6}$ can also be used to describe the transverse phonons.

From Eq. (38) we also find that the transverse part of the momentum density is given by

$$
g_{i}^{T}=\frac{1}{c_{\perp}^{2}} \epsilon_{i j} \partial_{j}\left(\partial_{\tau}^{2} A^{T T}\right) .
$$


Therefore the stress tensor has the nondiagonal contribution

$$
\pi_{i j}^{N D}=-\frac{1}{c_{\perp}^{2}} \epsilon_{i j}\left(\partial_{\tau}^{3} A^{T T}\right)=2 \rho c_{\perp}^{2} \epsilon_{i j} \vartheta_{6}
$$

and both the longitudinal as well as the transverse hydrodynamic equations in the solid phase can be summarized by

$$
\begin{gathered}
\frac{\partial \delta \rho}{\partial t}=-\nabla \cdot \vec{g} \\
\frac{\partial \vec{g}}{\partial t}=-c^{2} \nabla \delta \rho-\gamma_{\Delta} \nabla n_{\Delta}+2 \rho c_{\perp}^{2} \nabla \times \vartheta_{6}, \\
\frac{\partial \vartheta_{6}}{\partial t}=\frac{1}{2 \rho} \nabla \times \vec{g}, \\
\frac{\partial n_{\Delta}}{\partial t}=-\nabla \cdot \vec{J}, \\
\frac{\partial \vec{J}}{\partial t}=-c_{\Delta}^{2} \nabla n_{\Delta}+\gamma \nabla \delta \rho,
\end{gathered}
$$

after a transformation to real time, which in this case not only means that $\tau \rightarrow i t$ but also $\vec{g} \rightarrow i \vec{g}$ and $n_{\Delta} \rightarrow i n_{\Delta}$. Moreover, note that the constants $c, \gamma_{\Delta}, c_{\Delta}$, and $\gamma$ should here be interpreted as renormalized quantities which are determined in terms of the microscopic parameters of our gauge theory by a comparison with Eq. (35).

Now we are ready to discuss dissipation. In principle dissipation has already been included because there is a coupling between the net defect density $n_{\Delta}$ and the phonons. Hence if for example an interstitial were, in a discrete picture, to tunnel from one location to another there would be a 'shake up' of the phonon field. However, if we treat $n_{\Delta}$ as a smooth continuously varying field, the action in Eq. (34) is quadratic and the bilinear coupling $n_{\Delta} \partial^{2} \chi^{\prime}$ produces only mixing of the collective modes but no real dissipation. Therefore, we choose to include effective dissipation in the same manner as explained in detail by Zippelius, Halperin and Nelson.19 Using their notation we first of all add to the 
right-hand side of Eq. (44b) the terms $\left(\eta \nabla^{2} \vec{g}+\zeta \nabla(\nabla \cdot \vec{g})\right) / \rho$ associated with the dissipative part of the stress tensor $\pi_{i j}$ and representing viscous diffusion of the momentum density.

Next the question arises how we need to modify Eq. (440). This equation is a result of the fact that we have allowed the dislocations, and hence the interstitials and vacancies, to move freely through the lattice and used Eq. (34) for the free action of the defects. If the defects effectively experience friction (for example due to their interaction with the phonons), then it is more appropriate to add a Leggett friction term2 21 and use

$$
S_{0}\left[n_{\Delta}\right]=\int_{0}^{\hbar \beta} d \tau \int d \vec{x} \frac{E_{\Delta}}{2} n_{\Delta}\left(\frac{\rho}{\mu} \frac{\partial_{\tau}^{2}}{\partial^{2}}+i \frac{\rho}{\mu} \xi \partial_{\tau}+1\right) n_{\Delta}
$$

instead. The dispersions then indeed obey $\omega^{ \pm} \simeq \pm c_{\perp} k-i \xi k^{2} / 2$ at long wavelengths, and we must add the term $\xi \nabla(\nabla \cdot \vec{J})$ to the right-hand side of Eq. (44e). If we further assume that the transverse part of the defect current density behaves as in a gas and simply diffuses to zero with a diffusion constant $\kappa$, we obtain in total

$$
\begin{gathered}
\frac{\partial \delta \rho}{\partial t}=-\nabla \cdot \vec{g} \\
\frac{\partial \vec{g}}{\partial t}=-\frac{B}{\rho} \nabla \delta \rho-\gamma_{\Delta} \nabla n_{\Delta}+2 \rho c_{\perp}^{2} \nabla \times \vartheta_{6}+\frac{\eta}{\rho} \nabla^{2} \vec{g}+\frac{\zeta}{\rho} \nabla(\nabla \cdot \vec{g}), \\
\frac{\partial \vartheta_{6}}{\partial t}=\frac{1}{2 \rho} \nabla \times \vec{g}, \\
\frac{\partial n_{\Delta}}{\partial t}=-\nabla \cdot \vec{J}, \\
\frac{\partial \vec{J}}{\partial t}=-c_{\Delta}^{2} \nabla n_{\Delta}+\gamma \nabla \delta \rho+\kappa \nabla^{2} \vec{J}+\xi \nabla(\nabla \cdot \vec{J}),
\end{gathered}
$$

with $B=\rho \partial p /\left.\partial \rho\right|_{n_{\Delta}, T}=\rho c^{2}$ the appropriate isothermal bulk modulus in view of the fact that the pressure is a function of both the particle density as well as the net defect density. From thermodynamics we therefore also conclude that $\gamma_{\Delta}=\partial p /\left.\partial n_{\Delta}\right|_{\rho, T}$.

It is interesting to point out that these hydrodynamic equations differ from the results obtained by Zippelius, Halperin, and Nelson. In particular, their Eq. (3.32) differs from our Eq. (46e) and reads 


$$
\vec{J}=-\Gamma_{\Delta} \nabla\left(\frac{n_{\Delta}}{\chi_{\Delta}}-\gamma_{\Delta} \delta \rho\right)
$$

The difference can easily be traced back to the fact that Zippelius, Halperin, and Nelson assume on phenomenological grounds that the dynamics of the net defect density is purely diffusive. Indeed, we exactly reproduce their results if we use in our calculation a free action of the form

$$
S_{0}\left[n_{\Delta}\right]=\int_{0}^{\hbar \beta} d \tau \int d \vec{x} \frac{E_{\Delta}}{2} n_{\Delta}\left(\frac{i \partial_{\tau}}{D_{\Delta} \partial^{2}}+1\right) n_{\Delta}
$$

instead of Eq. (34). We can therefore consider the hydrodynamic equations of Zippelius, Halperin and Nelson as the overdamped (or classical) limit of our Eq. (46). Clearly, Kleinert's more microscopic approach does not lead to purely diffusive but in first instance to propagating behavior of the defects, which is appropriate for the quantum crystals of interest in Sec. IV. We now turn to the modification of the above results in the hexatic phase.

In the hexatic phase there are free dislocations present and $\chi^{\prime \prime}$ replaces $n_{\Delta}$ as the appropriate dynamical degree of freedom. To see most clearly how this comes about we will work perturbatively in $1 / E_{c}$. Up to first order in $1 / E_{c}$ the effective action for $\chi^{\prime \prime}$ is

$$
\int_{0}^{\hbar \beta} d \tau \int d \vec{x}\left\{\frac{1}{4 \mu(1+\nu)}\left(\partial^{2} \chi^{\prime \prime}\right)^{2}+\frac{1}{2 E_{c}}\left(\partial_{i} \chi^{\prime \prime}\right)\left(\frac{\rho}{\mu} \frac{\partial_{\tau}^{2}}{\partial^{2}}+1\right)^{-1}\left(\partial_{i} \chi^{\prime \prime}\right)\right\}
$$

which upon Fourier transformation displays two modes with $\omega^{2}=c_{\perp}^{2} \vec{k}^{2}+2 \mu^{2}(1+\nu) /\left(E_{c} \rho\right)$. So in the limit $E_{c} \rightarrow \infty$ (which physically means that we are looking at the nonhydrodynamic regime $\vec{k}^{2} \gg \mu / 2 E_{c}$ ) we approximately have

$$
\partial_{\tau}^{2} \chi^{\prime \prime}=-c_{\perp}^{2} \partial^{2} \chi^{\prime \prime}
$$

whereas the equations of motion for $A^{T L}$ and $A^{T T}$ are

$$
\partial_{\tau}^{2} A^{T L}=-c_{\|}^{2} \nabla^{2} A^{T L}+\frac{\nu}{1-\nu^{2}} \partial_{\tau} \chi^{\prime \prime}
$$

and 


$$
\partial_{\tau}^{2} A^{T T}=-c_{\perp}^{2} \nabla^{2} A^{T T}
$$

respectively. For the mass-density fluctuation we now find

$$
\delta \rho=-\frac{1}{c_{\|}^{2}} \partial_{\tau} \partial^{2} A^{T L}-\frac{\rho}{2(\mu+\lambda)} \partial^{2} \chi^{\prime \prime}
$$

and for the stress tensor

$$
\pi_{i j}^{D}=-\delta_{i j}\left\{\frac{1}{c_{\|}^{2}} \partial_{\tau}^{3} A^{T L}+\frac{\rho}{2(\mu+\lambda)} \partial_{\tau}^{2} \chi^{\prime \prime}\right\}=-\delta_{i j}\left\{c_{\|}^{2} \delta \rho+\frac{1}{2(1+\nu)} \partial^{2} \chi^{\prime \prime}\right\} .
$$

Putting all this together we obtain in first instance the following set of hydrodynamic equations for the hexatic phase

$$
\begin{gathered}
\partial_{\tau} \delta \rho=\nabla \cdot \vec{g}^{L}, \\
\partial_{\tau} \vec{g}^{L}=-\nabla \delta p=-c_{\|}^{2} \nabla \delta \rho-\frac{1}{2(1+\nu)} \nabla\left(\nabla^{2} \chi^{\prime \prime}\right) \\
\partial_{\tau}^{2} \chi^{\prime \prime}=-c_{\perp}^{2} \nabla^{2} \chi^{\prime \prime} \\
\partial_{\tau}^{2} A^{T T}=-c_{\perp}^{2} \nabla^{2} A^{T T} .
\end{gathered}
$$

Combining the longitudinal and transverse parts as before, this equals

$$
\begin{gathered}
\partial_{\tau} \delta \rho=\nabla \cdot \vec{g} \\
\partial_{\tau} \vec{g}=-c_{\|}^{2} \nabla \delta \rho-\frac{1}{2(1+\nu)} \nabla\left(\nabla^{2} \chi^{\prime \prime}\right)+2 \rho c_{\perp}^{2} \nabla \times \vartheta_{6} \\
\partial_{\tau}^{2} \chi^{\prime \prime}=-c_{\perp}^{2} \nabla^{2} \chi^{\prime \prime} \\
\partial_{\tau} \vartheta_{6}=-\frac{1}{2 \rho} \nabla \times \vec{g}
\end{gathered}
$$

and clearly reduces to the hydrodynamic equations for the ideal crystal if we put $\chi^{\prime \prime}=0$. 
We now have to consider how the above picture changes for a finite value of $E_{c}$. Here we can use the results of Sec. IIB. In the hydrodynamic regime $\vec{k}^{2} \ll \mu / 2 E_{c}$ we saw that the transverse speed of sound was renormalized to zero, because we found the quadratic (particle-like) dispersion $\omega^{2}=2 E_{c} \vec{k}^{4} / \rho$. As a result we have for the transverse part of the hydrodynamic equations

$$
\partial_{\tau}^{2} A^{T T}=\frac{2 E_{c}}{\rho} \partial^{4} A^{T T}
$$

which implies that in the right-hand side of Eq. (55b) we must replace $2 \rho c_{\perp}^{2} \nabla \times \vartheta_{6}$ by $-4 E_{c} \vec{e}_{z} \times \nabla\left(\nabla^{2} \vartheta_{6}\right)$. This gives

$$
\begin{gathered}
\partial_{\tau} \vec{g}^{T}=-4 E_{c} \vec{e}_{z} \times \nabla\left(\nabla^{2} \vartheta_{6}\right), \\
\partial_{\tau} \vartheta_{6}=-\frac{1}{2 \rho} \nabla \times \vec{g}^{T}
\end{gathered}
$$

which is in complete agreement with Zippelius, Halperin, and Nelson if we identify the Frank constant $K_{A}$ with $8 E_{c}$.

For the longitudinal part we need to analyze the dynamics of $\chi^{\prime \prime}$ and $A^{T L}$. A straightforward calculation shows that the effective action for these fields contains precisely the four modes already found in Sec. [IB]. The propagating modes with $\omega^{2}=2 \mu(1+\nu) \vec{k}^{2} / \rho$ obey $\chi^{\prime}=\chi^{\prime \prime}+\nu \partial_{\tau} A^{T L}=0$ and are therefore indeed associated with density fluctuations proportional to $\partial^{2} \chi^{\prime \prime}$. We thus need to use a renormalized longitudinal speed of sound equal to

$$
c=\sqrt{\frac{2 \mu(1+\nu)}{\rho}}=\sqrt{\frac{2 \mu}{\rho} \frac{2 \mu+2 \lambda}{2 \mu+\lambda}}
$$

that is always smaller than the longitudinal speed of sound in the solid phase. In fact, this actually exhausts the longitudinal hydrodynamic modes since the other modes in the effective action for $\chi^{\prime \prime}$ and $A^{T L}$ are gapped. As a result we now obtain in real time the following set of hydrodynamic equations for the hexatic phase

$$
\frac{\partial \delta \rho}{\partial t}=-\nabla \cdot \vec{g}
$$




$$
\begin{gathered}
\frac{\partial \vec{g}}{\partial t}=-c^{2} \nabla \delta \rho-\frac{K_{A}}{2} \vec{e}_{z} \times \nabla\left(\nabla^{2} \vartheta_{6}\right) \\
\frac{\partial \vartheta_{6}}{\partial t}=\frac{1}{2 \rho} \nabla \times \vec{g}
\end{gathered}
$$

not including dissipation.

To include dissipation we again follow Zippelius, Halperin and Nelson and add to the right-hand side of Eq. (59b) the terms $\left(\eta \nabla^{2} \vec{g}+\zeta \nabla(\nabla \cdot \vec{g})\right) / \rho$. However, we do not add the term $\kappa \nabla^{2} \vartheta_{6}$ to the right-hand side of Eq. (59c) because, just as in the solid phase, the dissipation of the transverse modes is already accounted for in the term $\eta \nabla^{2} \vec{g}$ that is added to the momentum equation. Put differently, a term of the form $\kappa \nabla^{2} \vartheta_{6}$ can be absorbed by an appropriate redefinition of $K_{A}$ and $\eta$. Again introducing the isothermal bulk modulus $B=\rho d p /\left.d \rho\right|_{T}=\rho c^{2}$ we then find

$$
\begin{gathered}
\frac{\partial \delta \rho}{\partial t}=-\nabla \cdot \vec{g} \\
\frac{\partial \vec{g}}{\partial t}=-\frac{B}{\rho} \nabla \delta \rho-\frac{K_{A}}{2} \vec{e}_{z} \times \nabla\left(\nabla^{2} \vartheta_{6}\right)+\frac{\eta}{\rho} \nabla^{2} \vec{g}+\frac{\zeta}{\rho} \nabla(\nabla \cdot \vec{g}) \\
\frac{\partial \vartheta_{6}}{\partial t}=\frac{1}{2 \rho} \nabla \times \vec{g}
\end{gathered}
$$

as our final result for the hexatic phase. Apart from the absence of a dissipative term in Eq. (60) it agrees with the findings of Zippelius, Halperin and Nelson and therefore contains the same mode structure as derived in that paper. For completeness sake, we mention however that the equations of motion for the hexatic order parameter $\vartheta_{6}$ can be derived from an effective action

$$
S^{e f f}\left[\vartheta_{6}\right]=\int_{0}^{\hbar \beta} d \tau \int d \vec{x} \frac{1}{2} \vartheta_{6}\left(4 \rho \frac{\partial_{\tau}^{2}}{\partial^{2}}+4 i \eta \partial_{\tau}-K_{A} \partial^{2}\right) \vartheta_{6}
$$

that can easily be understood physically: The first term on the right-hand side corresponds to the kinetic energy $\int d \vec{x} \rho\left(\partial_{\tau} \vec{u}\right)^{2} / 2$ of the displacement field. The second term is a Leggett friction term and the last term corresponds to the usual Frank energy, which is responsible for the fact that the hexatic to liquid transition is of the Kosterlitz-Thouless type. 


\section{HYDRODYNAMICS OF SUPERFLUID PHASES}

Having arrived at the hydrodynamic equations for the solid and hexatic phases, our next objective is to incorporate the effects of the additional hydrodynamic degree of freedom associated with the phase of the superfluid order parameter. Fortunately, from the microscopic theories developed for superfluid liquids 22 and gases 23 it is well known how we should proceed to obtain the hydrodynamic (two-fluid) equations for the superfluid phases starting from the equations for the normal phase. The procedure consists in principle of four steps. First, the total (average) density $\rho$ of the system is split up into a normal density $\rho_{n}$ and a superfluid density $\rho_{s}$. In general these densities are tensors of second rank, but for systems with hexagonal symmetry which are of interest here they are proportional to the identity $\delta_{i j}$ and can be considered as scalars. Second, the total momentum density $\vec{g}$ is similarly split up into a normal component $\rho_{n} \vec{v}_{n}$ and a superfluid component $\rho_{s} \vec{v}_{s}$ with a superfluid velocity that is purely longitudinal $\left(\nabla \times \vec{v}_{s}=0\right)$. Third, for an effectively isotropic system the dissipative terms in the momentum equation must be generalized to

$$
\eta \nabla^{2} \vec{v}_{n}+\zeta_{1} \frac{\rho_{s}}{\rho} \nabla\left(\nabla \cdot\left(\vec{v}_{s}-\vec{v}_{n}\right)\right)+\zeta_{2} \nabla\left(\nabla \cdot \vec{v}_{n}\right)
$$

Finally, we must add the dynamics of the superfluid velocity, which is basically determined from the Josephson relation and reads

$$
\frac{\partial \vec{v}_{s}}{\partial t}=-\frac{B}{\rho^{2}} \nabla \delta \rho+\zeta_{3} \frac{\rho_{s}}{\rho} \nabla\left(\nabla \cdot\left(\vec{v}_{s}-\vec{v}_{n}\right)\right)+\zeta_{4} \nabla\left(\nabla \cdot \vec{v}_{n}\right)
$$

where $B=\rho^{2} d \mu /\left.d \rho\right|_{T}$ is again the isothermal bulk modulus and $\mu$ is the chemical potential per unit mass. We again leave out temperature fluctuations since we are primarily interested in third-sound modes, for which these fluctuations are (at least qualitatively) unimportant.

\section{A. Supersolid}

To apply the above procedure to Eq. (46) we must realize that we are here in fact already dealing with a two-fluid hydrodynamics. We must therefore not only split up the 
total momentum density $\vec{g}$ into a normal and a superfluid component but also the net defect current, i.e. $\vec{J}=\overrightarrow{J_{n}}+\overrightarrow{J_{s}}$. Moreover, we have to account for the fact that the chemical potential, just like the pressure, is a function of the particle density and the net defect density. In this manner we arrive at the following hydrodynamic equations

$$
\begin{gathered}
\frac{\partial \delta \rho}{\partial t}=-\nabla \cdot \vec{g} \\
\frac{\partial \vec{g}}{\partial t}=-\frac{B}{\rho} \nabla \delta \rho-\gamma_{\Delta} \nabla n_{\Delta}+2 \rho c_{\perp}^{2} \nabla \times \vartheta_{6} \\
+\eta \nabla^{2} \vec{v}_{n}+\zeta_{1} \frac{\rho_{s}}{\rho} \nabla\left(\nabla \cdot\left(\vec{v}_{s}-\vec{v}_{n}\right)\right)+\zeta_{2} \nabla\left(\nabla \cdot \vec{v}_{n}\right) \\
\frac{\partial \vartheta_{6}}{\partial t}=\frac{1}{2 \rho} \nabla \times \vec{g}, \\
\frac{\partial \vec{v}_{s}}{\partial t}=-\frac{B}{\rho^{2}} \nabla \delta \rho+\beta_{\Delta} \nabla n_{\Delta}+\zeta_{3} \frac{\rho_{s}}{\rho} \nabla\left(\nabla \cdot\left(\vec{v}_{s}-\vec{v}_{n}\right)\right)+\zeta_{4} \nabla\left(\nabla \cdot \vec{v}_{n}\right) \\
\frac{\partial n_{\Delta}}{\partial t}=-\nabla \cdot \vec{J}, \\
\frac{\partial \vec{J}}{\partial t}=-c_{\Delta}^{2} \nabla n_{\Delta}+\gamma \nabla \delta \rho+\kappa \nabla^{2} \vec{J}_{n}+\xi_{1} \nabla\left(\nabla \cdot \vec{J}_{s}\right)+\xi_{2} \nabla\left(\nabla \cdot \vec{J}_{n}\right) \\
\frac{\partial t}{\vec{J}_{s}}=-\frac{B_{\Delta} \rho_{s}}{\rho^{2}} \nabla n_{\Delta}+\beta \rho_{s} \nabla \delta \rho+\xi_{3} \frac{\rho_{s}}{\rho} \nabla\left(\nabla \cdot \vec{J}_{s}\right)+\xi_{4} \nabla\left(\nabla \cdot \vec{J}_{n}\right)
\end{gathered}
$$

with $\beta_{\Delta}=-\partial \mu /\left.\partial n_{\Delta}\right|_{\rho, T}$. These represent nine equations for the nine unknown functions $\delta \rho, \vec{v}_{n}, \vec{v}_{s}, \vartheta_{6}, n_{\Delta}, \vec{J}_{n}$ and $\vec{J}_{s}$.

Although a complete analysis of the various hydrodynamic modes is now possible, we will consider here only the situation which is most relevant to experiments, namely that the normal part of the two-dimensional system is clamped to an underlying substrate. As a result we have $\vec{v}_{n}=\vec{J}_{n}=\overrightarrow{0}$ and Eqs. (63b) and (63t) determining the normal properties of the supersolid are no longer valid. The hydrodynamic equations therefore reduce to

$$
\frac{\partial^{2} \delta \rho}{\partial t^{2}}=\frac{B \rho_{s}}{\rho^{2}} \nabla^{2} \delta \rho-\beta_{\Delta} \rho_{s} \nabla^{2} n_{\Delta}+\zeta_{3} \frac{\rho_{s}}{\rho} \frac{\partial}{\partial t}\left(\nabla^{2} \delta \rho\right),
$$




$$
\frac{\partial^{2} n_{\Delta}}{\partial t^{2}}=\frac{B_{\Delta} \rho_{s}}{\rho^{2}} \nabla^{2} n_{\Delta}-\beta \rho_{s} \nabla^{2} \delta \rho+\xi_{3} \frac{\rho_{s}}{\rho} \frac{\partial}{\partial t}\left(\nabla^{2} n_{\Delta}\right)
$$

They contain two pairs of propagating modes, which in the limit of a small coupling constant $\beta \ll B B_{\Delta} / \beta_{\Delta} \rho^{4}$ essentially correspond to a pair of third-sound modes with $\delta \rho$ unequal to zero but a constant net defect density and a pair of modes with an oscillating net defect density.

One might have expected that the coupling of a superfluid density to a propagating defect density would have resulted in one pair of gapped excitations and one pair of gapless excitations instead. Consider, for example, two identical superfluid layers. If the layers are uncoupled the dynamics of the phases $\vartheta_{1}$ and $\vartheta_{2}$ of the layers is determined by the action

$$
S_{\text {layers }}\left[\vartheta_{1}, \vartheta_{2}\right]=\int_{0}^{\hbar \beta} d \tau \int d \vec{x}\left\{\frac{\rho^{2}}{2 B}\left(\partial_{\tau} \vartheta_{1}\right)^{2}+\frac{\rho_{s}}{2}\left(\nabla \vartheta_{1}\right)^{2}+\frac{\rho^{2}}{2 B}\left(\partial_{\tau} \vartheta_{2}\right)^{2}+\frac{\rho_{s}}{2}\left(\nabla \vartheta_{2}\right)^{2}\right\},
$$

which clearly has two pairs of gapless (third-sound) modes, one pair for each superfluid. If we couple the order parameters by allowing the particles to tunnel with an amplitude $-J / \rho$ from one layer to the other we must add a Josephson coupling

$$
S_{\text {tunnel }}\left[\vartheta_{1}, \vartheta_{2}\right]=-\int d \tau \int d \vec{x} J \cos \left(\vartheta_{1}-\vartheta_{2}\right)
$$

to this action. The hydrodynamics modes couple to form two in-phase and two out-of-phase excitations. The modes with $\vartheta_{1}$ and $\vartheta_{2}$ oscillating out of phase get gapped (i.e. $\omega^{2} \simeq B J / \rho^{2}$ for $\left.\vec{k}^{2} \ll J / \rho_{s}\right)$ and only the modes with $\vartheta_{1}$ and $\vartheta_{2}$ oscillating in phase remain gapless. Yet in Eq. (64) we find only gapless modes.

This paradox can be resolved by noting that we have made the standard assumption 19 that both the total number of particles and the net number of defects is conserved. Hence, after an atom has tunneled from a lattice site to the position of a vacancy, a new vacancy is created near the original site of the atom. The analogous process for the two coupled superfluid layers in not simply tunneling of individual atoms from one layer to another, but rather the exchange of a pair of atoms in different layers, returning the system to its original state. Such a process is not a Josephson coupling and therefore the modes remain gapless. 
The existence of separate conservation laws for the particle and defect density thus allows in principle two separate broken symmetries.

We also note in passing that the third-sound modes in Eq. (64) are not present in the hydrodynamic equations proposed by Andreev and Lifshitz 24 and considered in more detail by Liu.25 This is a result of the fact that these authors use a somewhat different physical picture for the supersolid phase: They assume that the superfluid current density is carried by (Bose condensed) defects and that the normal current density is solely due to lattice vibrations. Hence if we take $\vec{v}_{n}=\overrightarrow{0}$, which in their context means that $\partial \vec{u} / \partial t=\overrightarrow{0}$, only transport of defects is possible and only the latter two modes survive. However, as a consequence of their picture the hydrodynamic equations in the (normal) solid phase describe only longitudinal and transverse sound modes in an ideal lattice and do not include the effect of vacancies or interstitials. As explained above this is incorrect in principle and one should at least also allow for a normal current density due to the motion of defects. In addition, we have seen in Sec. III that even in the presence of defects the density fluctuations are equal to $-\rho u_{i i}^{P h y s}$. It is therefore perfectly reasonable that if there is superfluid mass transport possible in the solid, it can be caused both by the motion of defects and by lattice vibrations. Indeed, as an existence proof of this latter possibility we can for instance consider superfluid ${ }^{4} \mathrm{He}$ in a weak periodic and commensurate potential, which is clearly a supersolid without defects.

While it is generically possible to have both density and defect superfluid modes, we might expect however, for realistic films on realistic substrates, that in a supersolid it may be harder for particles to perform ring exchanges 15,26 than for vacancies to exchange positions. Thus, a priori, we might expect the effective superfluid stiffness for the density fluctuations to be smaller than that of the vacancies, perhaps to the point where the former is entirely absent. 


\section{B. Superhexatic}

We next turn to the superhexatic phase. In a similar manner as in Sec. IVA we obtain from Eq. (60) the full set of hydrodynamic equations

$$
\begin{gathered}
\frac{\partial \delta \rho}{\partial t}=-\nabla \cdot \vec{g} \\
\frac{\partial \vec{g}}{\partial t}=-\frac{B}{\rho} \nabla \delta \rho-\frac{K_{A}}{2} \vec{e}_{z} \times \nabla\left(\nabla^{2} \vartheta_{6}\right) \\
+\eta \nabla^{2} \vec{v}_{n}+\zeta_{1} \frac{\rho_{s}}{\rho} \nabla\left(\nabla \cdot\left(\vec{v}_{s}-\vec{v}_{n}\right)\right)+\zeta_{2} \nabla\left(\nabla \cdot \vec{v}_{n}\right) \\
\frac{\partial \vartheta_{6}}{\partial t}=\frac{1}{2 \rho} \nabla \times \vec{g} \\
\frac{\partial \vec{v}_{s}}{\partial t}=-\frac{B}{\rho^{2}} \nabla \delta \rho+\zeta_{3} \frac{\rho_{s}}{\rho} \nabla\left(\nabla \cdot\left(\vec{v}_{s}-\vec{v}_{n}\right)\right)+\zeta_{4} \nabla\left(\nabla \cdot \vec{v}_{n}\right)
\end{gathered}
$$

that leads to the usual two-fluid hydrodynamics of a superfluid if we omit Eq. (67d) and put $\vartheta_{6}=0$. Therefore these equations allow for first and second sound,27 and for a pair of transverse modes involving $\vec{v}_{n}^{T}$ and $\vartheta_{6}$ which are either dispersive or propagating depending on the sign of $\Delta=K_{A} / 4 \rho-\left(\eta / \rho_{n}\right)^{2}$ : If $\Delta \leq 0$ we have two purely dispersive modes with $\omega^{ \pm}=-i\left(\eta / \rho_{n} \pm \sqrt{-\Delta}\right) \vec{k}^{2} / 2$, wheras if $\Delta>0$ we have two propagating modes and the particle-like dispersion $\omega^{ \pm}= \pm \sqrt{\Delta} \vec{k}^{2} / 2-i\left(\eta / \rho_{n}\right) \vec{k}^{2} / 2$. However, considering again the case $\vec{v}_{n}=\overrightarrow{0}$ the hydrodynamic equations now simply reduce to

$$
\frac{\partial^{2} \delta \rho}{\partial t^{2}}=\frac{B \rho_{s}}{\rho^{2}} \nabla^{2} \delta \rho+\zeta_{3} \frac{\rho_{s}}{\rho} \frac{\partial}{\partial t}\left(\nabla^{2} \delta \rho\right),
$$

which contains only a pair of third-sound modes with the velocity $c_{3}=\sqrt{B \rho_{s} / \rho^{2}}$ and the diffusion constant $D_{3}=\zeta_{3} \rho_{s} / \rho$.

\section{CONCLUSIONS AND DISCUSSION}

In this paper we have derived the hydrodynamic equations for the supersolid and superhexatic phases of a neutral two-dimensional Bose fluid. For the supersolid these equations 
are rather complex, since they incorporate the effects of defect motion and lattice vibrations on both the normal and superfluid parts of the momentum density. Our physical picture for the influence on the superfluid part is roughly speaking that in a mean-field theory the condensate wavefunction $\Psi(\vec{x}, t)$ obeys the Schrödinger equation

$$
i \hbar \frac{\partial \Psi(\vec{x}, t)}{\partial t}=\left\{-\frac{\hbar^{2} \nabla^{2}}{2 m}+\int d \vec{x}^{\prime} V\left(\vec{x}-\vec{x}^{\prime}\right) n\left(\vec{x}^{\prime}, t\right)\right\} \Psi(\vec{x}, t)
$$

where $m$ is the mass of the Bose particles and $V\left(\vec{x}-\vec{x}^{\prime}\right)$ is their interaction. In addition, $n(\vec{x}, t)$ is the particle density which will be determined by an additional mean-field theory that, for a supersolid, shows the instability associated with the formation of a density wave. Hence the (thermal) average $\langle n(\vec{x}, t)\rangle$ is periodic in space and independent of time. As a result the condensate wavefunction is, if we neglect density fluctuations, also periodic and we have indeed both diagonal as well as off-diagonal long-range order. Fluctuations in the density, however, induce variations in the phase of the wavefunction and therefore in the superfluid velocity. Because these density fluctuations can be caused by both lattice vibrations and oscillations in the net defect density we conclude that both mechanisms can lead to superfluid motion. Together with the existence of a conservation law for the net number of defects, this explains from a more microscopic view why we found two thirdsound modes and two modes with an oscillatory net defect density in the case of a supersolid adsorbed onto a substrate.

For the superhexatic phase we have shown that the hexatic long-range order leads to an additional (as compared to the superfluid) hydrodynamic degree of freedom that affects only the transverse modes and is therefore at long wavelengths decoupled from the superfluid momentum density. This can also be understood from the above picture, since variations in the orientational order parameter $\vartheta_{6}$ do not lead to density fluctuations in first instance. As a result we find on a substrate only two third-sound modes and thus at the hydrodynamic level of description nothing to distinguish the superhexatic from the superfluid. Although this is in agreement with the experiments of Chen and Mochel, who indeed only observe one third-sound branch below the second critical temperature $T_{c}$, it is unfortunate for the 
purpose of suggesting a possible identification of the superhexatic phase. On the basis of our results we can, however, conclude that a more microscopic probe is needed if one wants to detect the orientational order present in a superhexatic helium film. In our opinion this appears to be an important, but also difficult experimental challenge.

Finally, we would also like to point out the possible relevance of our results to the recent experiments with bulk solid ${ }^{4} \mathrm{He} .{ }^{9}$ In these experiments Lengua and Goodkind observe at sufficiently high frequencies an additional (resonant) attenuation and velocity change of sound. Moreover, they notice that their data can be explained by a simple model of two coupled wave equations which turns out to be identical to the longitudonal part of our solid hydrodynamics derived in Sec. [II]. Because our two-dimensional hydrodynamics should be able to describe the propagation of sound perpendicular to the c-axis of hcp ${ }^{4} \mathrm{He}$, this confirms the conjecture of Lengua and Goodkind that the collective mode observed is associated with the motion of defects. For a more detailed discussion of the coupling between sound and the defects one should of course consider the fully three-dimensional situation and include the anisotropy of the hcp crystal. Work in this direction is in progress.

\section{ACKNOWLEDGMENTS}

This research was supported by Grant No. DMR-9502555 and DMR-9416906 from the National Science Foundation, the ESF Network on Quantum Fluids and Solids, the Swedish Natural Science Research Council and the Stichting voor Fundamenteel Onderzoek der Materie (FOM) which is financially supported by the Nederlandse Organisatie voor Wetenschappelijk Onderzoek (NWO). We thank Huug van Beelen, Henk van Beijeren, Michel Bijlsma, Reyer Jochemsen, and Anne van Otterlo for stimulating and helpful discussions. 


\section{REFERENCES}

${ }^{1}$ P. Kapitza, Nature 141, 74 (1938); and J.F. Allen and A.D. Misener, Nature 141, 75 (1938).

${ }^{2}$ I. Rudnick, Phys. Rev. Lett. 40, 1454 (1978); and D.J. Bishop and J.D. Reppy, Phys. Rev. Lett 40, 1727 (1978).

${ }^{3}$ M.T. Chen, J. Roesler, and J.M. Mochel, J. Low Temp. Phys. 89, 125 (1992); and J.M. Mochel and M.T. Chen, Phys. B 197, 278 (1994).

${ }^{4}$ For a different interpretation of these experiments see S.-C. Zhang, Phys. Rev. Lett. 27, 2142 (1993); and M. Gabay and A. Kapitulnik, Phys. Rev. Lett. 71, 2138 (1993).

${ }^{5}$ D.R. Nelson and J.M. Kosterlitz, Phys. Rev. Lett. 39, 1201 (1977).

${ }^{6}$ K. Mullen, H.T.C. Stoof, M. Wallin, S.M. Girvin, Phys. Rev. Lett. 72, 4013 (1994).

${ }^{7}$ D.R. Nelson and B.I. Halperin, Phys. Rev. B 19, 2457 (1979).

${ }^{8}$ A. van Otterlo and K.-H. Wagenblast, Phys. Rev. Lett. 72, 3598 (1994) and references therein.

${ }^{9}$ G.A. Lengua and J.M. Goodkind, J. Low. Temp. Phys. 79, 251 (1990).

${ }^{10}$ L. Balents (unpublished).

${ }^{11}$ S.C. Zhang, Int. J. Mod. Phys. B 6, 25 (1992).

${ }^{12}$ In this paper we will at times use the adjective 'superfluid' for those properties that superfluids, superhexatics and supersolids have in common, although we realize that hexatic and solid phases are not fluid.

${ }^{13}$ H. Kleinert, J. Phys. A 19, 1855 (1986).

${ }^{14}$ L.D. Landau and E.M. Lifshitz, Theory of Elasticity (Pergamon, New York, 1970). 
${ }^{15}$ H. Kleinert, Gauge Fields in Condensed Matter Physics (World Scientific, Singapore, 1989).

${ }^{16}$ F.R.N. Nabarro, Theory of Dislocations (Clarendon, New York, 1967), Chapter VII.

${ }^{17}$ H. Kleinert, Phys. Lett. A 91, 295 (1982).

${ }^{18}$ P.C. Martin, O. Parodi, and P.S. Pershan, Phys. Rev. A 6, 2401 (1972).

${ }^{19}$ A. Zippelius, B.I. Halperin, and D.R. Nelson, Phys. Rev. B 22, 2514 (1980).

${ }^{20}$ D.R. Nelson (unpublished).

${ }^{21}$ A.O. Caldeira and A.J. Leggett, Ann. Phys. 149, 374 (1983); and A.J. Leggett, S. Chakravarty, A.T. Dorsey, M.P.A. Fisher, A. Garg, and W. Zwerger, Rev. Mod. Phys. 59, 1 (1987).

22 P.C. Hohenberg and P.C. Martin, Ann. Phys. 34, 291 (1965).

${ }^{23}$ T.R. Kirkpatrick and J.R. Dorfman, J. Low. Temp. Phys. 58, 301 (1985); ibid. 58, 399 (1985).

${ }^{24}$ A.F. Andreev and L.M. Lifshitz, Sov. Phys. JETP 29, 1107 (1969).

${ }^{25}$ M. Liu, Phys. Rev. B 18, 1165 (1978).

${ }^{26}$ R.P. Feynman, Phys. Rev. 91, 1291 (1953).

${ }^{27}$ For a complete description of second sound one should of course also include temperature fluctuations. 


\section{FIGURES}

FIG. 1. A negative disclination in a hexagonal lattice. It is formed by removing a $60^{\circ}$ wedge from the lattice, and then distorting the lattice so that the open edges meet. A positive disclination (not shown) is formed by the insertion of a $60^{\circ}$ wedge of material into the lattice.

FIG. 2. A Volterra construction for a pair of dislocations: (a) a section of the two-dimensional lattice bounded by the loop $\mathcal{C}$ is removed and (b) the edges of the loop are pulled together to form the dashed line $\mathcal{L}$. The endpoints $\mathcal{P}$ of this line correspond to the positions of the dislocations.

FIG. 3. Dispersion curves for (a) the transverse and (b) the longitudinal modes in the hexatic phase. In both cases $\nu=1 / 2$.

FIG. 4. A Volterra construction for an interstitial in a hexagonal lattice: (a) three half-infinite lines of lattice points are removed (as indicated by the arrows) and (b) the lattice is distorted by drawing together the edges about the removed lines in such a manner that the crystal symmetry at large distances from the interstitial is restored. The removal of the three half-infinite lines is simply the Volterra construction of three dislocations. Thus an interstitial can be viewed as being made from three dislocations. A similar construction can be made for vacancies. 
Fig. 1 


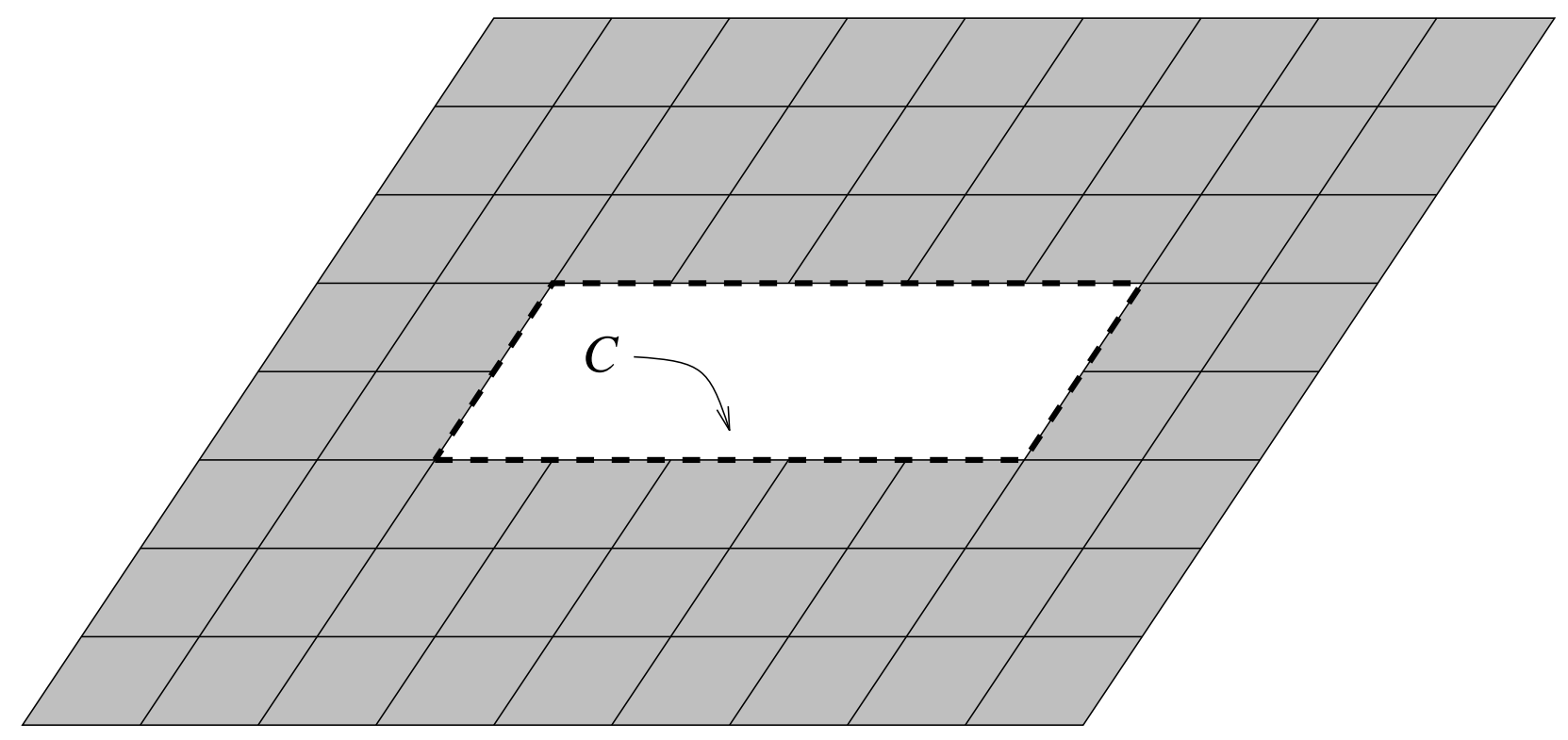

Fig. 2(a) 


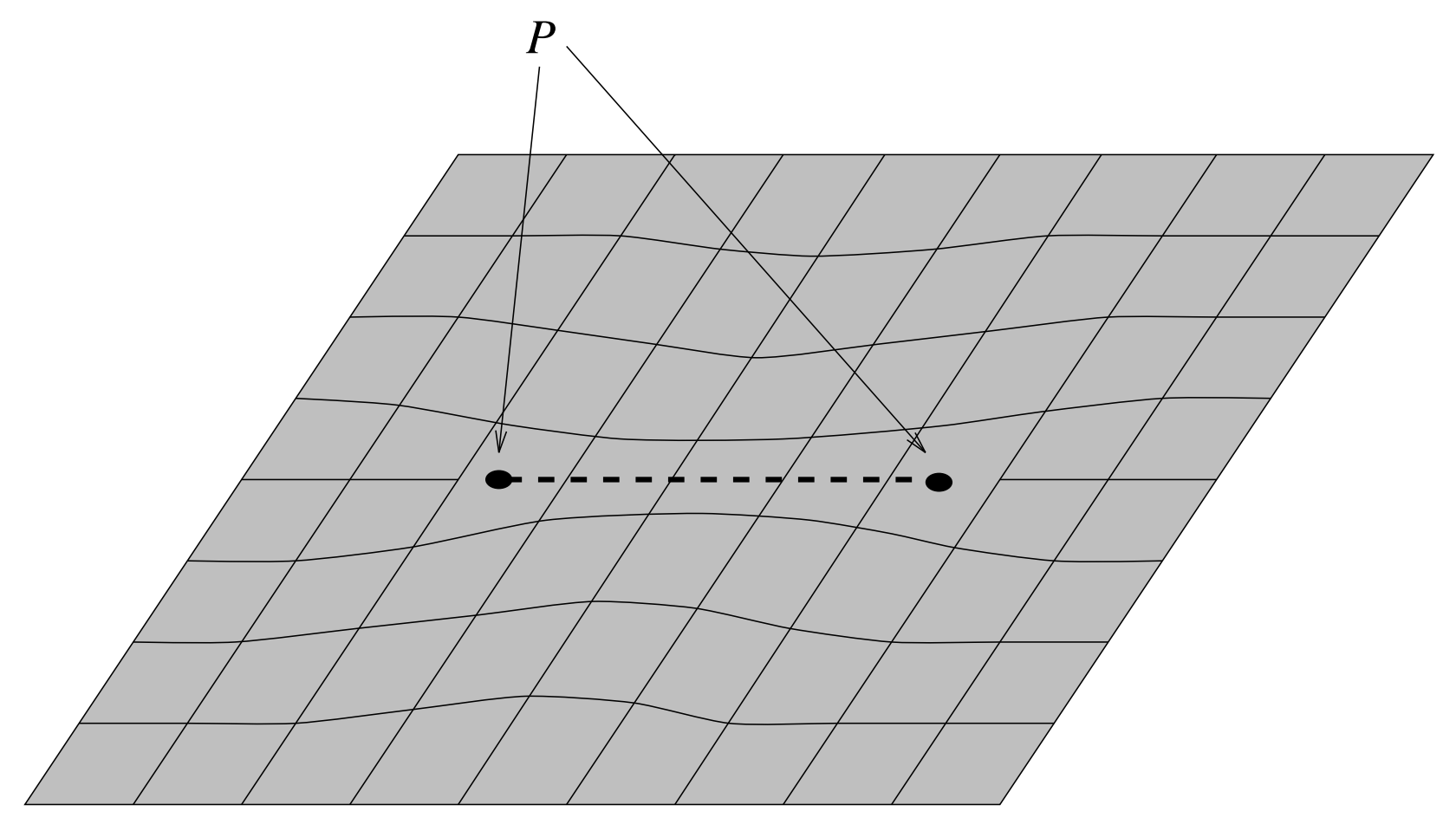

Fig. 2(b) 


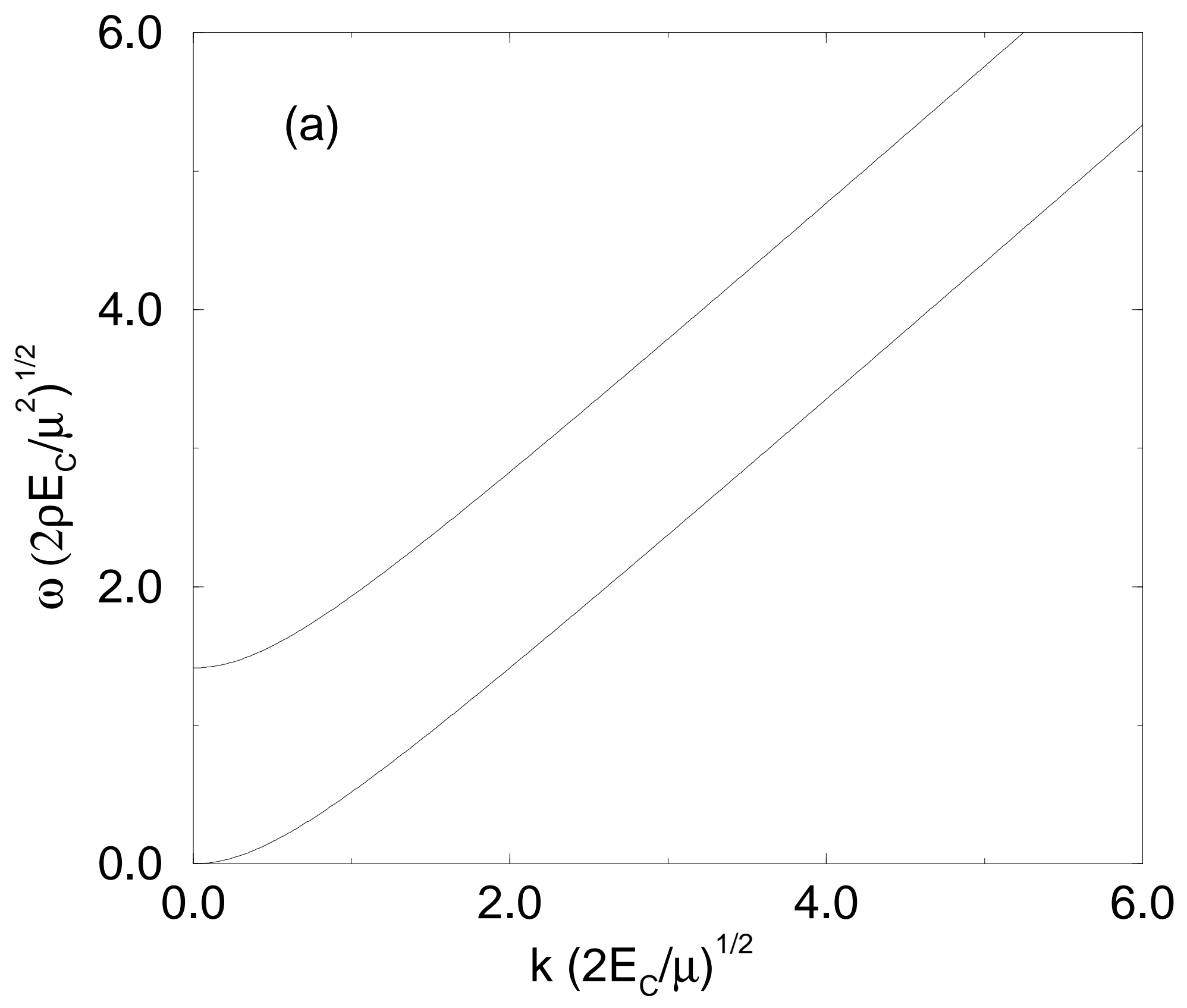




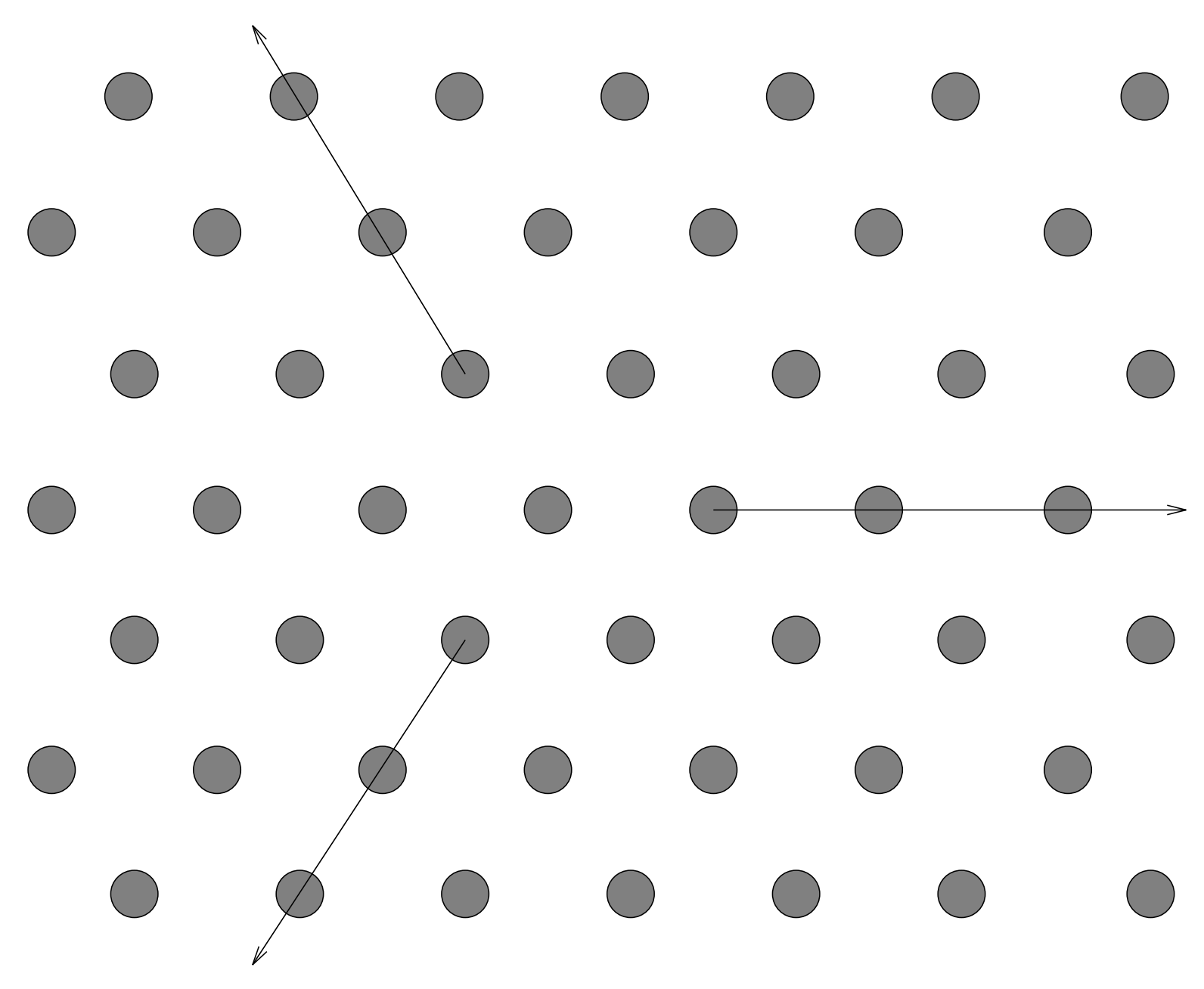

Fig. 4(a) 


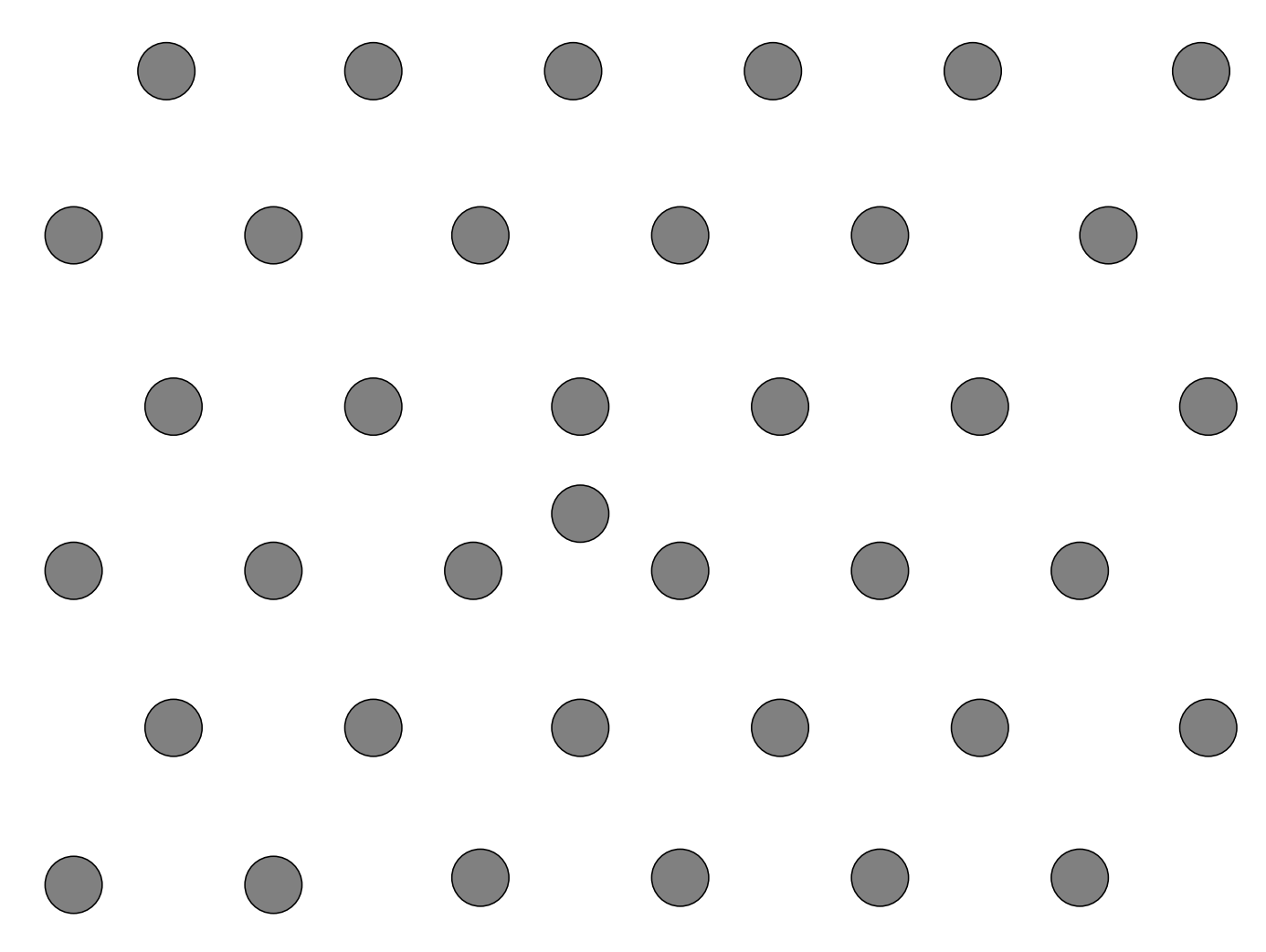

Fig. 4(b) 